УДК 581.6:582.47:674.8

\title{
КИНЕТИКА СОРБЦИИ КАТИОННЫХ КРАСИТЕЛЕЙ МОДИФИЦИРОВАННОЙ КОРОЙ ХВОЙНЫХ ДРЕВЕСНЫХ ПОРОД сиБИРи *
}

\author{
(C) А.В. Семенович** , С.Р. Лоскутов \\ Институт леса им. В.Н. Сукачева СО РАН, Академгородок, 50-28, \\ Красноярск, 660036 (Россия), e-mail: semenovich_a@ksc.krasn.ru
}

Исследован механизм кинетики сорбции катионов метиленового голубого из водного раствора модифицированной корой лиственницы сибирской (Larix sibirica L.), сосны обыкновенной (Pinus sylvestris L.) и пихты сибирской (Abies sibirica Ledeb.). Равновесие в распределении катионов красителя между раствором и сорбентами устанавливается через 8 ч после начала сорбции. Применение к экспериментальным кривым уравнений диффузионной и химической кинетики позволило установить следующее. Сорбционный процесс взаимодействия катионов красителя с функциональными группами модифицированной коры включает три последовательных стадии массопереноса сорбата на препаратах: диффузию молекул красителя из объема раствора через внешний диффузионный слой к поверхности частиц модифицированной коры (внешнедиффузионный массоперенос), диффузию молекул красителя в частицах модифицированной коры к активным центрам (внутридиффузионный массоперенос) и ионообменный процесс. Скорость сорбции лимитируется как диффузионными процессами, так и стадией взаимодействия катионов с функциональными группами сорбентов. С точки зрения кинетики исследовано влияние соотношения «масса сорбента : объем раствора» на извлечение катионов метиленового голубого из водного раствора.

Ключевые слова: модифицированная кора хвойных пород, метиленовый голубой, кинетика.

\section{Введение}

В настоящее время российские предприятия лесной отрасли накопили до 800 млн т древесной коры, около $30 \%$ от ее общей массы перерабатывается для получения технических продуктов (субстрата для изготовления удобрений, мульчи и др.), значительные объемы вывозят в отвалы, чем наносится вред окружающей среде. Перспективное направление утилизации коры - получение сорбентов различного назначения, что оправдано следующими причинами: возобновимые и достаточные запасы сырья, возможность размещения производства вблизи источника сырья, непосредственное использование отходов производства для получения нового продукта, возможность дальнейшего использования отработанного сорбента.

Применение необработанной коры для извлечения загрязнителей из сточных вод приводит к вымыванию из нее органических соединений и к необходимости устранения вторичного загрязнения очищаемого стока. Для повышения ценности коры как сорбента предложено химическое модифицирование ее поверхности [1]. Суть модифицирования заключается в переводе абсорбционно-активных соединений в водонерастворимое состояние и/или их иммобилизации, а также экстрагировании неактивных окрашивающих соединений.

Семенович Анжелика Владимировна - младший научный сотрудник лаборатории физико-химической биологии древесных растений, кандидат химических наук, e-mail: semenovich_a@ksc.krasn.ru

Лоскутов Сергей Реджинальдович - заместитель директоpa, доктор химических наук, e-mail: 1sr@ksc.krasn.ru
Сорбционная способность модифицированной коры хвойных древесных пород Сибири по отношению к катионным красителям в статических условиях исследована нами ранее [2]. Вызывает интерес изучение кинетики сорбции. Это по-

\footnotetext{
* Данная статья имеет электронный дополнительный материал (приложение), который доступен читателям на сайте журнала. DOI: 10.14258/jeprm.201504912s

Автор, с которым следует вести переписку.
} 
зволяет установить скорость достижения равновесия при сорбции, механизм сорбции, совокупность элементарных стадий сорбции, определить стадию, лимитирующую скорость сорбционного процесса в целом и оценить целесообразность применения нового сорбента в реальных условиях.

Цель настоящей работы - изучение кинетики сорбции катионного красителя метиленового голубого из водного раствора модифицированной корой хвойных древесных пород Сибири.

\section{Экспериментальная часть}

В качестве исходного сырья использовали кору лиственницы сибирской (Larix sibirica Ledeb.), coсны обыкновенной (Pinus sylvestris L.) и пихты сибирской (Abies sibirica Ledeb). Отбор сырья производили в зоне Красноярской лесостепи (Красноярский край, Средняя Сибирь). Кору измельчали, просеивали и отделяли фракцию 0,5-1,0 мм. Химическое модифицирование коры осуществляли по методу [1]: катализатор - $3 \% \mathrm{HNO}_{3}$, продолжительность модифицирования - 15 мин, температура $-50{ }^{\circ} \mathrm{C}$.

Для изучения кинетики сорбции красителя метиленового голубого (МГ) сорбентом точные навески сорбента (0,01-0,2 г) размещали в колбах Эрленмейера емкостью 0,05 дм $^{3}$, заливали водными растворами МГ с концентрацией 240 мг/дм³ , объемом 0,025 дм³. Через равные промежутки времени (от 1 до 1440 мин) определяли равновесную концентрацию МГ в растворе фотометрическим методом с помощью колориметра-нефелометра фотоэлектрического (ФЭК-56 М, при $\lambda=400 \pm 10$ нм, светофильтр №3) [3].

Количество сорбированного красителя при времени $t$ вычисляли по формуле:

$$
\mathrm{S}_{\mathrm{t}}=\frac{\left(C_{0}-C_{t}\right) \cdot V}{m}
$$

где $S_{t}$ - количество МГ, поглощенного сорбентом, мг/г в момент времени $t$, мин; $C_{o}-$ концентрация красителя в исходном растворе, мг/дм ${ }^{3} ; C_{t}$ - равновесная концентрация МГ в растворе, мг/дм ${ }^{3}$ в момент времени $t ; V-$ объем раствора МГ, взятого для контактирования с сорбентом, дм³ ; $m$ - масса воздушно-сухого сорбента, г.

Кинетические кривые сорбции аппроксимировали с помощью уравнений диффузионной и химической кинетики (табл. 1 в электронном приложении). Для оценки качества подбора линейной функции использовали коэффициент детерминированности $\left(\mathrm{R}^{2}\right)$ и корреляции, (R); сумму квадратов ошибок (SSE); сумму абсолютных ошибок (SAE); среднюю относительную ошибку (COO); относительную стандартную ошибку среднего (ARS); сумму стандартного отклонения (CCO); сумму квадратов отклонений от их среднего значения (СКО), вычисленные в программной среде Microsoft Excel.

\section{Обсуждение результатов}

В результате модифицирования коры лиственницы, сосны и пихты получены твердые сыпучие сорбенты, при контакте которых с раствором катионного красителя метиленового голубого вторичного загрязнения очищаемого раствора не происходит, при этом модифицированная кора (МК) обладает относительно высокой сорбционной способностью по отношению к МГ (табл. 1). При исходной концентрации раствора МГ, равной 240 мг/дм³, способность сорбентов извлекать катионы из раствора снижается в ряду: модифицированная кора пихты > модифицированная кора сосны > модифицированная кора лиственницы. Различие сорбционной способности сорбентов по отношению к катионам МГ может объясняться различием природы и концентрации функциональных групп, ответственных за взаимодействие с катионами, и различным соотношением микро- и мезопор сорбентов.

Для выяснения механизма взаимодействия катионов МГ с функциональными группами МК были получены кинетические кривые сорбции (рис. 1). Как видно, в начальный период сорбции количество сорбирующегося МГ препаратами увеличивается очень быстро, затем, по мере приближения к равновесию, увеличение замедляется. Резкий подъем скорости в начале сорбции может указывать как на быстрый массоперенос молекул красителя из раствора к активным центрам сорбента [10], так и на быстрое химическое связывание крупных органических катионов красителя с активными центрами на его поверхности [11]. Равновесие в распределении катионов красителя между раствором и сорбентами устанавливается через 8 ч после начала сорбции. 
Для аналитического выражения кинетических кривых был выбран метод, заключающийся в обработке кинетических кривых уравнениями диффузионной кинетики (внешнедиффузионной и внутридиффузионной) и уравнениями химической кинетики (псевдопервого порядка, псевдовторого порядка). Результаты графической обработки опытных данных в соответствующих координатах представлены на рисунках 2-4. Кинетические параметры, полученные применением теоретических уравнений к данным, иллюстрирует таблица 1 (оценка пригодности моделей для описания кинетических кривых приведена в табл. 2 электронного приложения).

По построенным кривым и по величине коэффициентов детерминации, которые варьируют от 0,833 до 0,987, видно, что все модели в той или иной степени удовлетворительно описывают кинетику сорбции катионов МГ модифицированной корой. При этом наблюдается некоторый разброс экспериментальных точек от аналитических графиков. Это указывает на то, что сорбционное взаимодействие катионов МГ и функциональных групп модифицированной коры осложнено множеством побочных процессов.

Рис. 1. Кинетические кривые сорбции катионов МГ из водного раствора с концентрацией 240 мг/дм ${ }^{3}$ модифицированной корой. Соотношение «масса сорбента - объем раствора» составляет $1: 250$ г/см ${ }^{3}$. По оси ординат: $S_{t}-$ количество сорбированного катиона МГ на единицу массы сорбента в момент времени $t$, мг/г; по оси ординат: время, мин

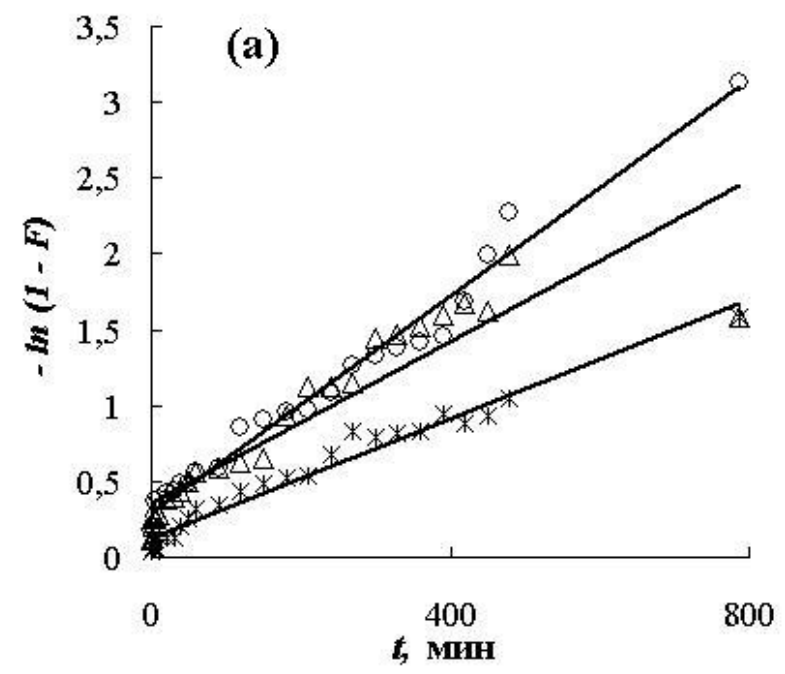

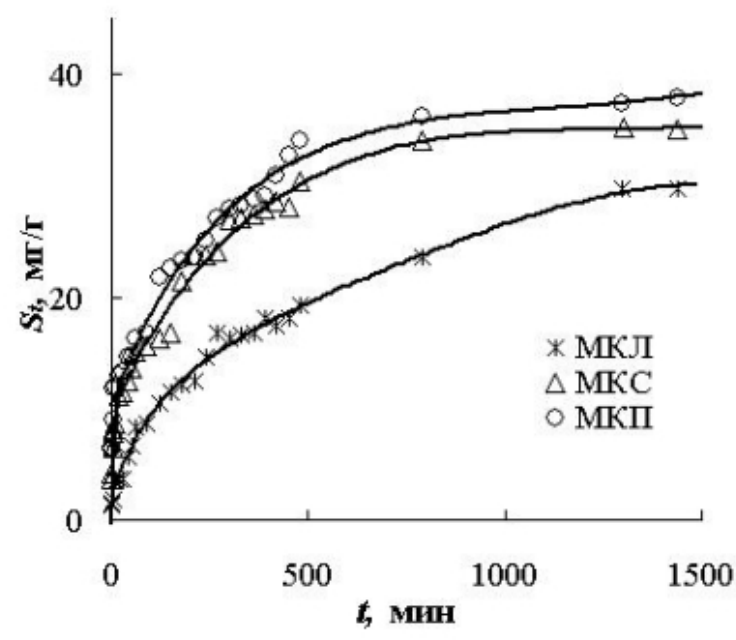

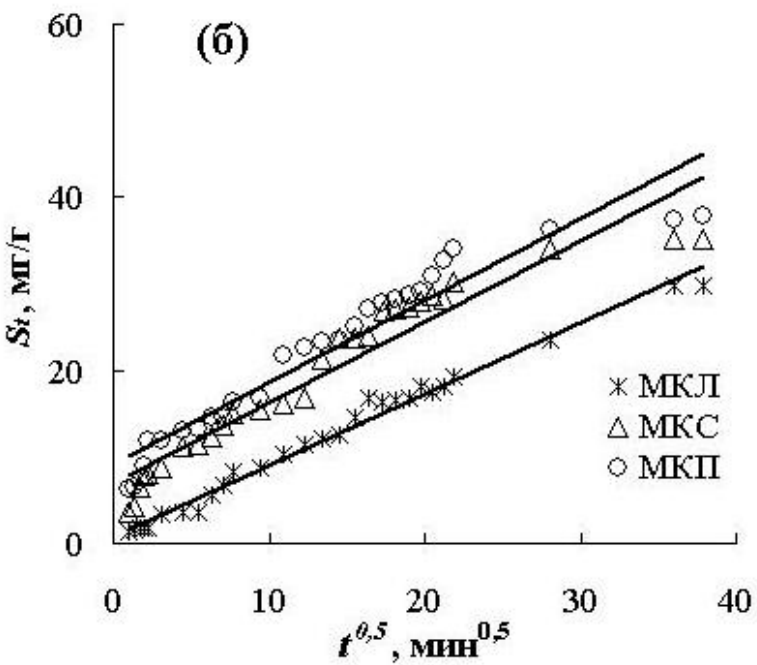

Рис. 2. Анаморфозы кинетических кривых сорбции МГ модифицированной корой лиственницы, сосны и пихты в координатах уравнений, описывающих (а) внешнедиффузионные, по оси абсцисс: $t-$ время, мин; по оси ординат: $-\ln (1-F)$, где $F$ - степень достижения равновесия в системе, $F=S_{t} / S_{e}$, где $S_{t}-$ количество сорбированного катиона МГ на единицу массы сорбента в момент времени $t$, мг/г, $S_{e}-$ количество сорбированного катиона МГ на единицу массы сорбента при равновесии, мг/г; (б) внутридиффузионные процессы, по оси абсцисс: $t^{0,5}$ - период полураспада, мин ${ }^{0,5}$; по оси ординат: $S_{t}$ - количество сорбированного катиона МГ на единицу массы сорбента в момент времени $t$, мг/г. Концентрация катионов МГ в исходном водном растворе - 240 мг/дм ${ }^{3}$, соотношение «масса сорбента - объем раствора» - $1: 250$ г/см ${ }^{3}$ 

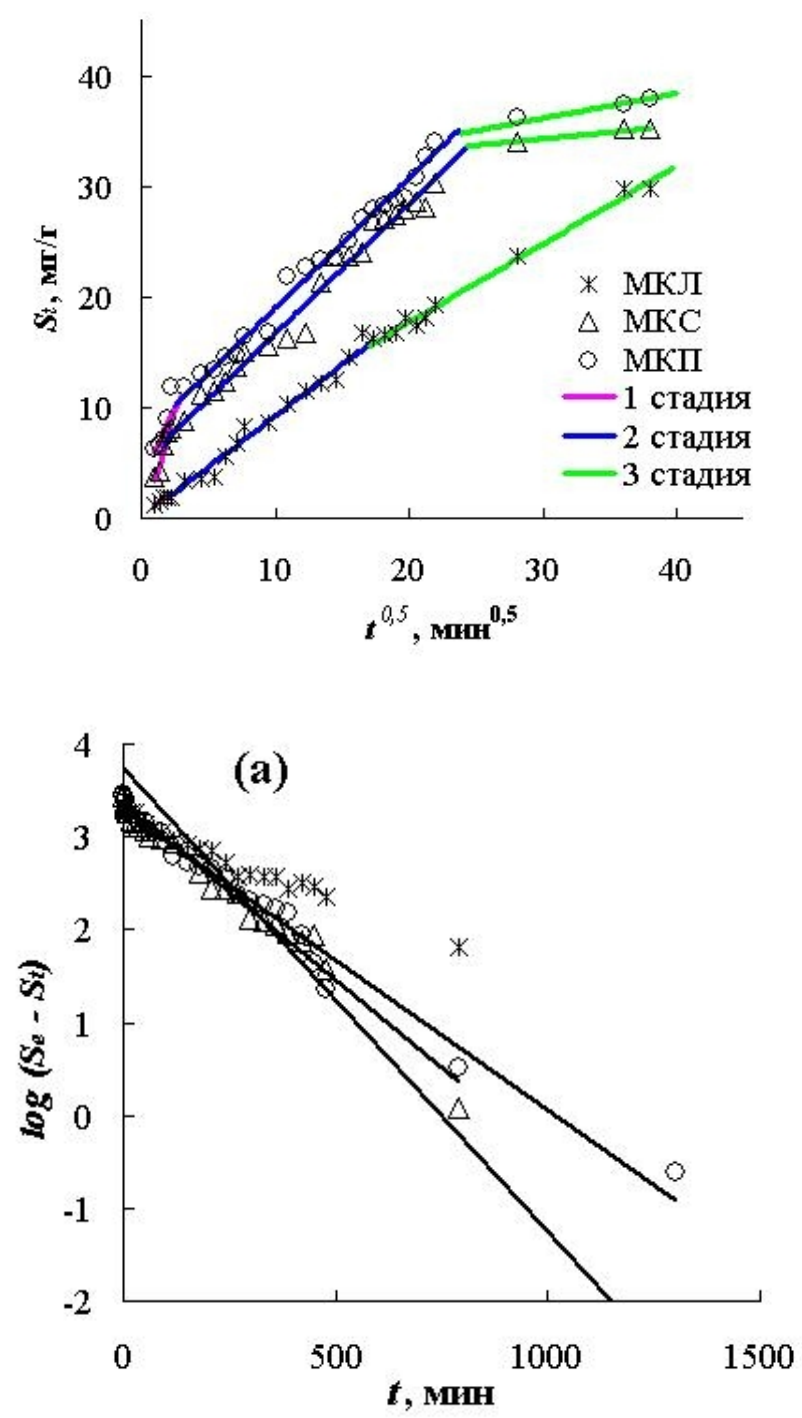

Рис. 3. Мультилинейная зависимость $S_{t}=f\left(t^{0,5}\right)$ для сорбции катионов метиленового голубого из водных растворов модифицированной корой при $20{ }^{\circ} \mathrm{C}$. По оси абсцисс: $t^{0,5}-$ период полураспада, мин $^{0,5}$; по оси ординат: $S_{t}-$ количество сорбированного катиона МГ на единицу массы сорбента в момент времени $t$, мг/г

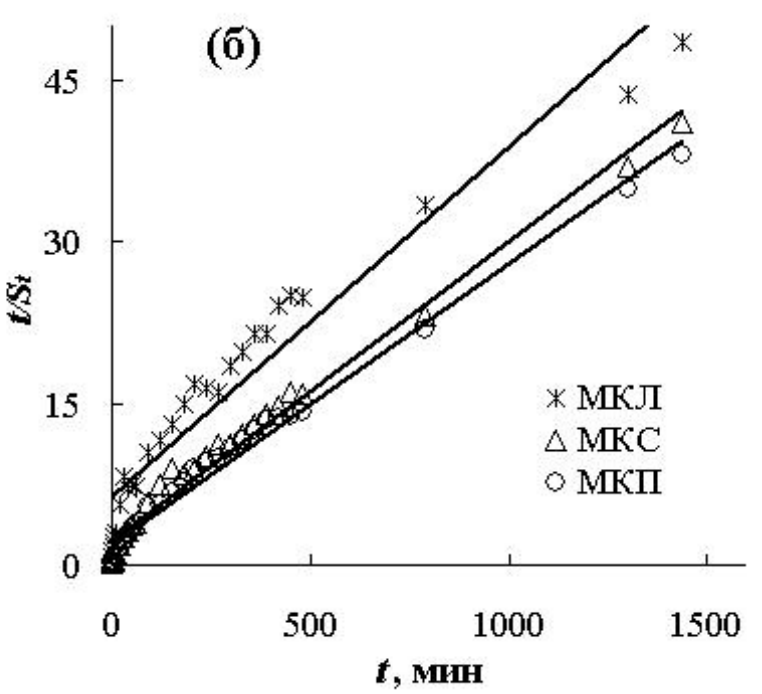

Рис. 4. Анаморфозы кинетических кривых сорбции МГ из водных растворов модифицированной корой лиственницы, сосны и пихты в координатах уравнений, описывающих реакции (а) псевдопервого порядка, по оси абсцисс: $t$ - время, мин; по оси ординат: $\log \left(S_{e}-S_{t}\right)$ - логарифм разности количества сорбированного катиона МГ на единицу массы сорбента при равновесии, $\left(S_{e}\right)$ мг/Г и количества сорбированного катиона МГ на единицу массы сорбента в момент времени $t,\left(S_{t}\right)$ мг/г; (б) псевдовторого порядка, по оси абсцисс: $t$ - время, мин; по оси ординат: $t / S_{t}$ - отношение времени, мин к количеству сорбированного катиона МГ на единицу массы сорбента в момент времени $t$, мг/г. Концентрация катионов МГ в исходном водном растворе - 240 мг/дм³ ${ }^{3}$ соотношение «масса сорбента - объем раствора $\mathrm{M \Gamma »}-1: 250, \Gamma / \mathrm{cm}^{3}$.

Все кинетические кривые описываются уравнением для внешнедиффузионных процессов (рис. 2а), однако зависимости $-\ln (1-F)=f(t)$ не выходят из начала координат и становятся прямолинейными спустя 3-5 мин после начала опыта. Данный факт свидетельствует о том, что сорбционный процесс на модифицированной коре протекает в смешаннодиффузионом режиме, т.е. контролируется одновременно внешней и внутренней диффузией.

Для проверки соответствия кинетических кривых модели внутридиффузионной кинетики была проведена обработка кинетических кривых в координатах $S_{t}-t^{0,5}$. Результаты показывают (рис. 2б), что по мере увеличения продолжительности контактирования модифицированной коры с красителем количество сорбированных катионов МГ возрастает пропорционально величине $t^{0,5}$ вплоть до достижения равновесия. Полученные зависимости являются мультилинейными и не выходят из начала координат (рис. 3), что свидетельствует о многостадийном взаимодействии сорбата с сорбентом. 
Таблица 1. Параметры сорбции МГ модифицированной корой, полученные применением кинетических уравнений к опытным данным

\begin{tabular}{|c|c|c|c|}
\hline Параметры $^{1}$ & $\mathrm{MK}^{2}$ & MKC & МКП \\
\hline$S_{e}, \mathrm{M \Gamma} / \Gamma$ & 29,73 & 35,11 & 37,87 \\
\hline \multicolumn{4}{|c|}{ Модель внутридиффузионной кинетики } \\
\hline$K_{i(1)}, \mathrm{MГ \Gamma}^{-1} \mathrm{мин}^{-0,5}$ & 0,83 & 1,24 & 0,71 \\
\hline$A_{(1)}$ & 0,41 & 2,45 & 5,52 \\
\hline$K_{i(2)}, \mathrm{мг \Gamma}^{-1}$ мин $^{-0,5}$ & 0,93 & 1,19 & 1,19 \\
\hline$A_{(2)}$ & $-0,04$ & 4,72 & 6,87 \\
\hline 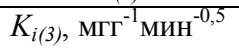 & 0,71 & 0,39 & 0,22 \\
\hline$A_{(3)}$ & 3,54 & 21,07 & 29,35 \\
\hline \multicolumn{4}{|c|}{ Модель псевдопервого порядка } \\
\hline$K_{f}$, мин $^{-1}$ & $4,60 \cdot 10^{-3}$ & $8,75 \cdot 10^{-3}$ & $8,29 \cdot 10^{-3}$ \\
\hline$S_{e}, \mathrm{M \Gamma} / \Gamma$ & 26,23 & 28,33 & 28,48 \\
\hline \multicolumn{4}{|c|}{ Модель псевдовторого порядка } \\
\hline$K_{s}$, Гмг ${ }^{-1} \mathrm{Mин}^{-1}$ & $0,17 \cdot 10^{-3}$ & $0,34 \cdot 10^{-3}$ & $0,35 \cdot 10^{-3}$ \\
\hline$S_{e}, \mathrm{M \Gamma} / \Gamma$ & 30,86 & 36,10 & 38,46 \\
\hline
\end{tabular}

Примечания: ${ }^{1}$ где $S_{e}$ - количество сорбированного катиона МГ на единицу массы сорбента при равновесии, мг/г; $K_{i(1)}$, $K_{i(2)}, K_{i(3)}$ - константы скорости внутренней диффузии, мг $\cdot \Gamma^{-1} \cdot$ мин $^{-0,5}$ на 1,2 и 3 стадии соответственно; $A_{(1)}, A_{(2)}, A_{(3)}-$ отрезок, отсекаемый графической зависимостью $S_{t}=f\left(t^{0,5}\right)$ при его экстраполяции до оси ординат, пропорционален величине толщины пленки, окружающей частицу сорбента; $K_{f}-$ константа скорости псевдопервого порядка, мин ${ }^{-1} ; K_{s}-$ константа скорости псевдовторого порядка, $Г \cdot \mathrm{M \Gamma}^{-1} \cdot \mathrm{мин}^{-1}$.

${ }^{2}$ МКЛ, МКС, МКП - модифицированная кора лиственницы, сосны и пихты соответственно.

В процессе обмена, происходящего между функциональными группами модифицированной коры и комплексными катионами красителя, можно выделить три последовательных стадии массопереноса сорбата на препаратах.

Первый крутой участок характеризует диффузию молекул красителя из объема раствора через внешний диффузионный слой (неподвижную водяную пленку, образующуюся при контакте сорбентов с раствором на наружной поверхности омываемой частицы модифицированной коры) к поверхности частиц модифицированной коры (внешнедиффузионный массоперенос). Второй участок относится к диффузии молекул красителя в частицах модифицированной коры к активным центрам (внутридиффузионный массоперенос). Вытесняющий катион МГ перемещается сквозь слой уже адсорбированных поверхностью сорбента катионов красителя вглубь частиц модифицированной коры через систему пор и капилляров. Третий, завершающий пологий участок отражает ионообменный процесс.

В соответствии со скоростью диффузии сорбата в зависимости от стадий его массопереноса сорбенты можно условно разделить на две группы. В первую группу входит МКС, во вторую - МКЛ и МКП.

Кинетика сорбции катионов МГ препаратом МКС характеризуется интенсивной диффузией молекул красителя из объема раствора к твердой фазе сорбента. Движение молекул за счет диффузии ничем не ограничено. Катионы МГ легко диффундируют через внешний диффузионный слой к поверхности сорбента, молекулы движутся с наибольшими скоростями, толщина водяной пленки, окружающей частицы сорбента, невелика, что облегчает прохождение катионов МГ к поверхности частиц МКС. Причинами торможения диффузии на второй стадии массопереноса могут являться затруднения, вызванные столкновением молекул, которые могут происходить из-за соизмеримых радиусов равновесных ассоциатов МГ и большинства пор сорбента и/или различной доступностью функциональных групп в препарате МКС.

Иная ситуация складывается при сорбции красителя препаратами второй группы. Диффузия катионов МГ в поры МКЛ и МКП происходила с большей скоростью, чем диффузия из разбавленного и недостаточно интенсивно перемешиваемого водного раствора к внешней поверхности сорбентов.

Третья стадия массопереноса катионов МГ из раствора в сорбенты как первой, так и второй группы отличается относительно низкой скоростью диффузии. Движущей силой диффузионного процесса является разность концентраций переходящего вещества МГ во взаимодействующих фазах: остаточная концентрация красителя в водном растворе минимальна, равновесная концентрация красителя внутри частиц сорбентов высока. Разность концентраций по мере приближения системы к равновесию чрезвычайно мала, вследствие чего катионы МГ диффундируют значительно медленнее.

Известно, что скорость всего многостадийного процесса сорбции определяется скоростью наиболее медленной стадии. При исследовании сорбции катионов МГ модифицированной корой выявлено, что наи- 
более медленной стадией является стадия химической реакции между сорбируемыми катионами МГ и функциональными группами модифицированной коры, что возможно, в случае если обменивающиеся катионы предрасположены к гидролизу и/или ионный обмен осложнен ионными реакциями [4]. Поскольку лимитирующая стадия - это стадия химической реакции, которая может протекать как внутри частиц модифицированной коры, так и на ее поверхности, то скорость сорбционного процесса в целом будет зависеть от концентрации катионов и температуры. Процесс протекает в кинетической области реагирования и должен описываться уравнениями химической кинетики.

Для выявления вклада химической стадии в скорость сорбции катионов МГ сорбентами кинетические кривые были обработаны с помощью уравнений химической кинетики. Кинетические кривые для сорбционных систем в координатах соответствующих уравнений иллюстрирует рисунок 4.

Имеющиеся сравнительные данные (рис. 4, табл. 1) свидетельствуют о хорошей корреляции между фактическими и предсказанными обеими моделями химической кинетики зависимостями. Различие между значениями $S_{e}$, полученными опытным путем и вычисленными при помощи обоих уравнений, значительно меньше для всех сорбционных систем при использовании модели псевдовторого порядка. Соответствие опытных данных кинетическому уравнению псевдовторого порядка указывает на то, что взаимодействие между сорбатом и функциональной группой сорбента подчиняется закону действующих масс для реакций второго порядка (скорость взаимодействия пропорциональна произведению концентраций двух реагирующих веществ), компоненты взаимодействуют друг с другом в соотношении 1:1 [10]. Подобные результаты получены при изучении кинетики сорбции МГ опилками дуба, обработанными щелочью и измельченными и отмытыми горячей водой листьями дерева гуавы Psidium guajava [5; 12].

Однако анализ ряда ошибок (табл. 2, электронное приложение) показал, что для всех сорбционных систем имеет преимущества уравнение псевдопервого порядка (минимальные значения SSE, SAE, COO, $\mathrm{ARS}, \mathrm{CCO}, \mathrm{CKO}$, максимальные значения $\mathrm{R}^{2}$ и R). Данный факт свидетельствует о том, что сорбционный процесс протекает в условиях относительно малой концентрации одного из реагентов по отношению к другому, и скорость взаимодействия зависит от концентрации только этого вещества. Диффузия предшествует сорбции.

На рисунке 5 изображены кинетические кривые начального этапа сорбции катионов МГ модифицированной корой пихты (первые пять минут контакта сорбента с сорбатом) при использовании различных соотношений «масса сорбента : объем раствора». Большинство кривых отражают мгновенный процесс взаимодействия красителя с поверхностью сорбентов. Следует отметить одну особенность формы кинетической кривой, полученной при использовании наибольшего соотношения «масса сорбента : объем раствора МГ» равного $1: 2500$ (кривая 1). Как видно, катионы начинают сорбироваться сорбентом лишь после 1 мин от начала контакта. Для этой кривой, по сравнению с другими, наблюдается минимальное увеличение количества сорбирующегося красителя сорбентом в течение первых пяти минут их контакта. Однако при достижении равновесия величина сорбции катионов МГ модифицированной корой пихты оказалась максимальной - 83,33 мг/г (табл. 2).

Таблица 2. Параметры сорбции МГ модифицированной корой пихты, полученные применением уравнений к опытным данным при различных соотношениях «масса сорбента : объем раствора»

\begin{tabular}{|c|c|c|c|c|c|c|}
\hline \multirow{2}{*}{ Параметры* } & \multicolumn{6}{|c|}{ 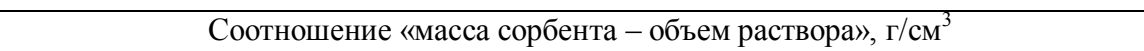 } \\
\hline & $1: 2500$ & $1: 1000$ & $1: 500$ & $1: 250$ & $1: 166$ & $1: 125$ \\
\hline$S_{e}, \mathrm{M \Gamma} / \Gamma$ & 83,33 & 64,90 & 49,76 & 37,87 & 28,29 & 24,26 \\
\hline \multicolumn{7}{|c|}{ Модель внешнедиффузионной кинетики } \\
\hline$R^{2}$ & 0,58 & 0,90 & 0,91 & 0,93 & 0,86 & 0,91 \\
\hline \multicolumn{7}{|c|}{ Модель внутридиффузионной кинетики } \\
\hline$K_{i}$, мгг $^{-1}$ мин $^{-0,5}$ & 4,32 & 8,29 & 11,18 & 4,83 & 7,24 & 6,43 \\
\hline$R^{2}$ & 0,67 & 0,94 & 0,92 & 0,88 & 0,91 & 0,93 \\
\hline \multicolumn{7}{|c|}{ Модель псевдопервого порядка } \\
\hline$K_{f}$, мин $^{-1}$ & 0,04 & 0,13 & 0,26 & 0,15 & 0,35 & 0,39 \\
\hline$R^{2}$ & 0,58 & 0,90 & 0,91 & 0,93 & 0,86 & 0,91 \\
\hline \multicolumn{7}{|c|}{ Модель псевдовторого порядка } \\
\hline$K_{s}$, гмг ${ }^{-1}$ мин $^{-1}$ & 0,40 & $7,39 \cdot 10^{-5}$ & $1,25 \cdot 10^{-6}$ & 0,02 & 0,01 & 0,02 \\
\hline$R^{2}$ & 0,99 & 0,01 & 0,0003 & 0,80 & 0,92 & 0,96 \\
\hline
\end{tabular}

*параметры те же, что и в табл. 1 
Рис. 5. Влияние соотношения «масса сорбента : объем раствора» на сорбцию МГ из раствора с концентраций 240 мг/дм³ в первые 5 мин контакта сорбента с сорбатом. Обозначения: кривая 1 - соотношение составляет 1 г МК : $2500 \mathrm{~cm}^{3}$ раствора МГ; кривая $2-1: 1000$, кривая $3-1: 500$, кривая $4-1: 250$, кривая $5-1$ : 166, кривая $6-1: 125$. По оси абсцисс: время, мин; по оси ординат: $S_{t}-$ количество сорбированного катиона МГ на единицу массы сорбента в момент времени $t$, мг/Г

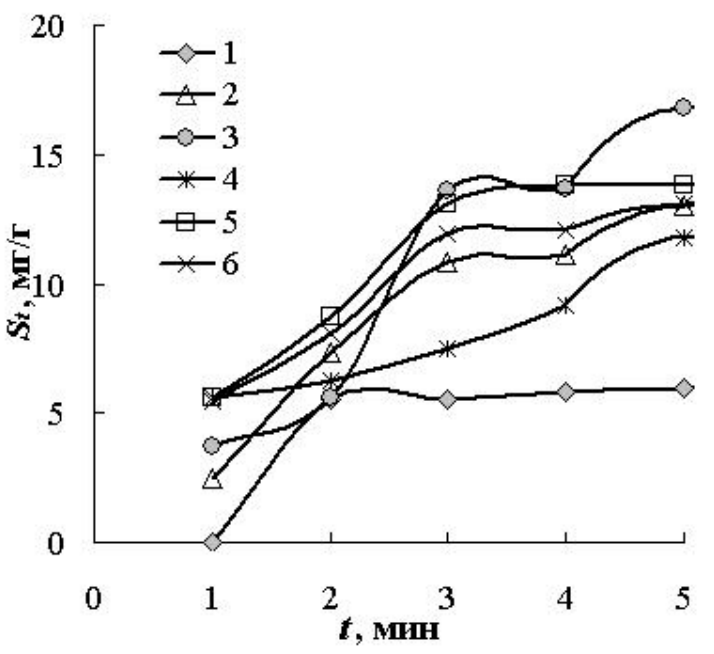

Существование начального индукционного периода, вероятно, связано с тормозящими процессами, происходящими вблизи поверхности сорбента. Извлечение катионов МГ происходило из водного раствора с относительно высокой концентрацией красителя. Можно предположить, что указанный промежуток времени затрачивается на конкуренцию гидратированных катионов с ассоциированными молекулами красителя за относительно малое, но более доступное для сорбции количество свободных активных центров сорбента, находящихся как на его поверхности, так и в объеме. Некоторые мицеллы красителя не могут удержаться активными центрами поверхности сорбента, так как мицеллы имеют слоистую структуру и большую длину ассоциированных молекул. Поэтому они связываются с подобными мицеллами МГ, содержащимися в водном растворе. В этом случае сорбционный процесс будет происходить с формированием мономолекулярного слоя, на который впоследствии «зацепляются» мицеллы МГ.

Проверка соответствия кинетических кривых уравнениям диффузионной и химической кинетики показала, что уже в первые 5 мин контакта сорбента с сорбатом используемое соотношение «масса сорбента : объем раствора» оказывает влияние на кинетику сорбции МГ из раствора с концентрацией 240 мг/дм ${ }^{3}$ модифицированной корой (кинетические параметры приведены в табл. 2). В случае использования для извлечения катионов МГ из водного раствора модифицированной корой максимального соотношения «масса сорбента : объем раствора» (кривая 1) и минимальных соотношений (кривые 5 и 6) наилучшим образом описывает опытные данные уравнение псевдовторого порядка. Однако при применении средних отношений (кривые 2 и 3) преимущество имеет модель внутридиффузионной кинетики.

\section{Bыводbl}

Перспективным направлением утилизации многотоннажного отхода окорки древесины является химическое модифицирование коры Larix sibirica Ledeb, Pinus sylvestris L. и Abies sibirica L. для получения сорбентов, способных извлекать крупные органические катионы красителя метиленового голубого из сточных вод. Сорбционная способность модифицированной коры по отношению к катионам МГ варьирует от 24,26 до 83,33 мг/г в зависимости от типа исходного сырья и соотношения «масса сорбента : объем рас-

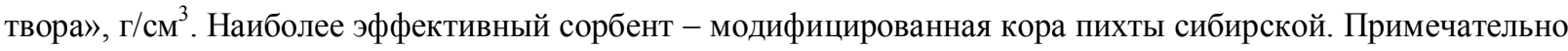
и то, что модифицированию кора может подвергаться без разделения ее по породам.

Механизм сорбции катионов МГ модифицированной корой достаточно сложен. Анализ кинетических кривых показал, что при контакте сорбента с сорбатом в начальный период сорбции (продолжительность процесса $\leq 5$ мин) кинетика сорбции зависит от интенсивности внешнего массопереноса. Затем по мере увеличения степени заполнения модифицированной коры катионами красителя усиливается влияние на общую скорость сорбции внутридиффузионного массопереноса, и лимитирующей стадией становится внутренняя диффузия. Вносит свой вклад в общую скорость сорбции и стадия взаимодействия катионов МГ с функциональными группами сорбента, обусловленная образованием комплексов в фазе сорбента. Дифференцировать вклад диффузии и химической стадии путем применения кинетических уравнений затруднительно, не исключено наличие их синергетического воздействия на скорость сорбции. 


\section{Список литературы}

1. Palma G., Freer J., Baeza J. Removal of metal ions by modified Pinus radiata bark and tannins from water solution // Water Research. 2003. Vol. 37, N20. Pp. 4974-4980.

2. Семенович А.В., Лоскутов С.Р. Адсорбция катионных красителей модифицированной корой хвойных древесных пород // Химия растительного сырья. 2004. №3. С. 121-125.

3. ГОСТ 4453-74. Уголь активный осветляющий, древесный, порошкообразный. Технические условия. М., 1976. $10 \mathrm{c}$.

4. Лакиза Н.В. Равновесие и кинетика процессов разделения и концентрирования ионов переходных металлов карбоксиэтилированными полисилоксанами : автореф. дис. ... канд. хим. наук. Екатеринбург, 2007. 24 с.

5. Abd El-Latif M.M., Ibrahim A.M., El-Kady M.F. Adsorption Equilibrium, kinetics and thermodynamics of methylene blue from aqueous solutions using biopolymer oak sawdust composite // Journal of American Science. 2010. Vol. 6, N6. Pp. 267-283.

6. Argun M.E., Dursun S. A new approach to modification of natural adsorbent for heavy metal adsorption // Bioresource Technology. 2008. Vol. 99, N7. Pp. 2516-2527.

7. Waranusantigul P., Pokethitiyook P., Kruatrachue M., Upatham E.S. Kinetics of basic dye (methylene blue) biosorption by giant duckweed (Spirodela polyrrhiza) // Environmental Pollution. 2003. Vol. 125. Pp. 385-392.

8. Mohan D., Pittman C.U., Bricka J.M., Smith F., Yancey B., Mohammad J., Steele P.H., Alexandre-Franco M.F., Gómez-Serrano V., Gong H. Sorption of arsenic, cadmium and lead by chars produced from fast pyrolysis of wood and bark during bio-oil production // Journal of Colloid and Interface Science. 2007. Vol. 310, N1. Pp. 57-73.

9. Ünlü N., Ersoz M. Adsorption characteristics of heavy metal ions onto a low cost biopolymeric sorbent from aqueous solutions // Journal of Hazardous Materials. 2006. Vol. 136, N2. Pp. 272-280.

10. Ho Y.-S., Ng J.C.Y., McKay G.M. Kinetics of pollutant sorption by biosorbents: review // Separation Purification Methods. 2000. Vol. 29, N2. Pp. 189-232.

11. Lazaridis N.K., Karapantsios D., Georgantas D. Kinetic analysis for the removal of a reactive dye from aqueous solution onto hydrotalcite by adsorption // Water Research. 2003. Vol. 37. Pp. 3023-3033.

12. Ponnusami V., Vikram S., Srivastava S.N. Guava (Psidium guajava) leaf powder: Novel adsorbent for removal of methylene blue from aqueous solution // Journal of Hazardous Materials. 2008. Vol. 152, N1. Pp. 276-286.

Поступило в редакиию 20 ноября 2015 г.

После переработки 9 декабря 2015 г. 
Semenovich A.V. ${ }^{*}$, Loskutov S.R. THE KINETICS OF SORPTION OF CATIONIC DYES MODIFIED BARK CONIFEROUS TREE SPECIES OF SIBERIA

V.N. Sukachev Institute of Forest SB RAS, Akademgorodok, 50-28, Krasnoyarsk, 660036 (Russia),

e-mail: semenovich_a@ksc.krasn.ru

The mechanism of sorption kinetic of methylene blue cations from an aqueous solution by the modified bark of Siberian larch (Larix sibirica L.), Scotch pine (Pinus sylvestris L.) and Siberian fir (Abies sibirica Ledeb.) was studied. Adsorption equilibrium in the distribution of the dye cations between the solution and the sorbent is reached in 8 hours after the beginning of sorption. Application to experimental curves of the equations of diffusive and chemical kinetics allowed establishing the following. Sorption process of interaction of cations of dye with functional groups of the modified bark includes three consecutive stages of a mass transfer of sorbate on preparations: diffusion of molecules of dye from solution volume through an external diffusive layer to a surface of particles of the modified bark (the external mass transfer), diffusion of molecules of dye in particles of the modified bark to the active centers (an internal mass transfer) and ion-exchange process. The sorption rate was limited by both diffusion processes and the stage of interaction of cations with functional groups of sorbents. From the point of view of kinetics influence of a ratio «adsorbent mass:liquid volume» on extraction of cations methylene blue of water solution is investigated.

Keywords: modified bark coniferous tree species, methylene blue, kinetic.

\section{References}

1. $\quad$ Palma G., Freer J., Baeza J. Water Research, 2003, vol. 37, no. 20, pp. 4974-4980.

2. Semenovich A.V., Loskutov S.R. Khimiia rastitel'nogo syr'ia, 2004, no. 3, pp. 121-125. (in Russ.).

3. GOST 4453-74. Ugol' aktivnyi osvetliaiushchii, drevesnyi, poroshkoobraznyi. Tekhnicheskie usloviia. [State Standard 4453-74. Coal active lightening, woody, powdery. technical conditions]. Moscow, 1976, 10 p. (in Russ.).

4. Lakiza N.V. Ravnovesie $i$ kinetika protsessov razdeleniia $i$ kontsentrirovaniia ionov perekhodnykh metallov karboksietilirovannymi polisiloksanami: avtoref. dis. ... kand. khim. nauk. [Equilibrium and kinetics of separation and concentration of transition metal ions karboksietilirovannymi polysiloxanes: the dissertation author's candidate of chemical science]. Ekaterinburg, 2007, 24 p. (in Russ.).

5. Abd El-Latif M.M., Ibrahim A.M., El-Kady M.F. Journal of American Science, 2010, vol. 6, no. 6, pp. $267-283$.

6. Argun M.E., Dursun S. Bioresource Technology, 2008, vol. 99, no. 7, pp. 2516-2527.

7. Waranusantigul P., Pokethitiyook P., Kruatrachue M., Upatham E.S. Environmental Pollution, 2003, vol. 125, pp. 385-392.

8. Mohan D., Pittman C.U., Bricka J.M., Smith F., Yancey B., Mohammad J., Steele P.H., Alexandre-Franco M.F., Gómez-Serrano V., Gong H. Journal of Colloid and Interface Science, 2007, vol. 310, no. 1, pp. 57-73.

9. Ünlü N., Ersoz M. Journal of Hazardous Materials, 2006, vol. 136, no. 2, pp. 272-280.

10. Ho Y.-S., Ng J.C.Y., McKay G.M. Separation Purification Methods, 2000, vol. 29, no. 2, pp. 189-232.

11. Lazaridis N.K., Karapantsios D., Georgantas D. Water Research, 2003, vol. 37, pp. 3023-3033.

12. Ponnusami V., Vikram S., Srivastava S.N. Journal of Hazardous Materials, 2008, vol. 152, no. 1, pp. 276-286.

Received November 20, 2015

Revised December 9, 2015

\footnotetext{
* Corresponding author.
} 
